University of South Carolina

Scholar Commons

$12-2009$

\title{
How Values in Scientific Discovery and Pursuit Alter Theory Appraisal
}

Kevin C. Elliott

University of South Carolina - Columbia, elliotkc@mailbox.sc.edu

Daniel J. McKaughan

Boston College, daniel.mckaughan@bc.edu

Follow this and additional works at: https://scholarcommons.sc.edu/phil_facpub

Part of the Philosophy Commons

\section{Recommended Citation}

(C) 2009 by the Philosophy of Science Association http://www.philsci.org/

This Article is brought to you by the Philosophy, Department of at Scholar Commons. It has been accepted for inclusion in Faculty Publications by an authorized administrator of Scholar Commons. For more information, please contact digres@mailbox.sc.edu. 


\title{
How Values in Scientific Discovery and Pursuit Alter Theory Appraisal
}

\author{
Kevin C. Elliott and Daniel J. McKaughan ${ }^{\dagger}$
}

Philosophers of science readily acknowledge that nonepistemic values influence the discovery and pursuit of scientific theories, but many tend to regard these influences as epistemically uninteresting. The present paper challenges this position by identifying three avenues through which nonepistemic values associated with discovery and pursuit in contemporary pollution research influence theory appraisal: (1) by guiding the choice of questions and research projects, (2) by altering experimental design, and (3) by affecting the creation and further investigation of theories or hypotheses. This analysis indicates that the effects of these values are sufficiently complex and epistemically significant to merit further attention.

1. Introduction. Philosophers now commonly acknowledge that values of an "epistemic" or "cognitive" sort inevitably play a role in scientific reasoning (Kuhn 1977; Laudan 1984). Moreover, there are a number of ways in which nonepistemic values also appear to play an appropriate role in science. Helen Longino (1990, 83-85) describes four points at which values might interact with scientific practice, and it is arguably unproblematic for nonepistemic values to influence the first three (see also Machamer and Wolters 2004). The first point of potential interaction involves the choice of projects and problems that researchers study. The second point is the development of policy decisions about how to apply scientific knowledge (e.g., deciding whether to adopt nuclear energy generation). The third point is the limitation of research methodologies based on moral concerns (e.g., prohibitions on testing the toxicity of pesticides on human beings). The fourth point of potential interaction, where many thinkers would seek to exclude nonepistemic values, concerns the evaluation and justification of scientific claims.

$\dagger$ To contact the authors, please write to: Kevin Elliott, Department of Philosophy, University of South Carolina, Columbia, SC 29208; e-mail: ke@sc.edu. Daniel McKaughan, Department of Philosophy, Boston College, Chestnut Hill, MA 02467; e-mail: daniel.mckaughan@bc.edu.

Philosophy of Science, 76 (December 2009) pp. 598-611. 0031-8248/2009/7605-0020\$10.00 Copyright 2009 by the Philosophy of Science Association. All rights reserved. 
Most recent discussions of values in science have focused on the fourth aspect of scientific practice. Arguments for allowing nonepistemic values to play a role in scientific reasoning commonly appeal either to the underdetermination of theory choice by purely epistemic considerations (see, e.g., Longino 1990; Howard 2006) or to the "inductive risk" of accepting a false theory that has nonepistemic consequences (see, e.g., Rudner 1953; Douglas 2000). But these arguments are controversial, in part because the extent of theoretical underdetermination is contested (Giere 2003; Dorato 2004) and because nonepistemic considerations are arguably irrelevant to a theory's truth value (as opposed to assessments of a theory's usefulness as a practical basis for action; Giere 2003; Mitchell 2004).

Despite the widespread acknowledgment that value influences in Longino's first three categories are "undeniable and omnipresent" (Dorato 2004, 56), they are often dismissed as epistemically insignificant. What sort of legitimate effects could they have on theory appraisal? Consider, for example, Stephanie Ruphy's analysis:

"Contextual" (i.e. external, non-"truth-seeking") values may shape scientific knowledge to the extent that they play a role in the definition of research programs, in the choice of questions deemed scientifically interesting, in the way scientific results might be applied, etc. This contextualization of the goals of science does not in itself threaten objectivity. More epistemologically challenging is the distinct charge that the very content of scientific knowledge is shaped by contextual values. (2006, 189-190; italics in original).

Mauro Dorato similarly asserts that the influence of nonepistemic values on Longino's first point of interaction "is clearly not sufficient, by itself, to deprive the social or the natural sciences of their value-free character from a cognitive point of view" (2004, 56; italics in original).

The present paper challenges such quick dismissals by seeking to show how nonepistemic values that enter at Longino's first point of interaction (namely, values associated with the choice of research projects) can wind up having significant implications for the fourth, more contested, arena of theory appraisal. In other words, we will focus on activities associated with scientific "discovery" or what Laudan (1977) calls "pursuit," including (among other things) the choice of research projects and questions, the generation and development of theories or hypotheses, the collection of data, and the decision to analyze particular theories or hypotheses rather than others. Using case studies from contemporary research on toxic pollutants, the paper examines three avenues through which nonepistemic values associated with discovery and pursuit influence scientific theory appraisal. This analysis shows that these values ultimately have significant epistemic effects that are easily overlooked. 
2. Overview of Three Avenues of Influence. A very straightforward principle underlies all three of the avenues through which nonepistemic values associated with scientific discovery and pursuit affect theory appraisal. The degree of evidential support for a theory clearly depends both on the array of available theories and on the set of data at hand. Therefore, to the extent that the nonepistemic values associated with discovery and pursuit influence the available theories and data, they affect theory appraisal. This point is vividly illustrated by reflecting on murder mysteries. Consider first the difference that varying the set of available theories can make to appraisal. The bumbling sheriff knows the butler had a motive and no alibi and takes the evidence to support the claim that the butler did it. Indeed, clever fiction writers take pains to tell their stories in such a way as to ensure that the probability of the butler hypothesis given the evidence is very high. Along comes the smart detective with an alternative hypothesis, and it becomes clear that the maid hypothesis is vastly superior to the butler hypothesis given the available evidence. Changes in the available data make a similar difference. Say that we have two alternative hypotheses - the butler did it and the maid did it — and the evidence clearly points to the butler. But the only reason is that the maid has been hiding the crucial piece of evidence. Once the smart detective finds the bloody knife with her fingerprints on it, it is clear that the maid is guilty. ${ }^{1}$

This might still appear to be an epistemically uninteresting sense in which the values associated with discovery and pursuit affect theory appraisal. Perhaps no one doubts that nonepistemic values influence the data and theories available to scientists; instead, the crucial epistemic point (one might argue) is whether the evidential relationship between a particular theory and a specific body of evidence is affected by nonepistemic values. For the sake of argument, we do not deny in this paper that the evidential relationship between a theory and a body of data (assuming a fixed set of background assumptions) should be free of nonepistemic influences. We insist, however, that the epistemically interesting aspects of theory appraisal include more than just the evidential relationships between specific data and theories. In particular, if nonepistemic values associated with scientific discovery and pursuit cause scientists to accept or believe one theory rather than another, we take that influence to be epistemically significant. An objector might retort, along the lines of Richard Jeffrey (1956), that scientists should not be in the business of accepting hypotheses. We contend that this is an overly restrictive view of scientific activity, and it may limit the ability of scientists to make socially relevant 


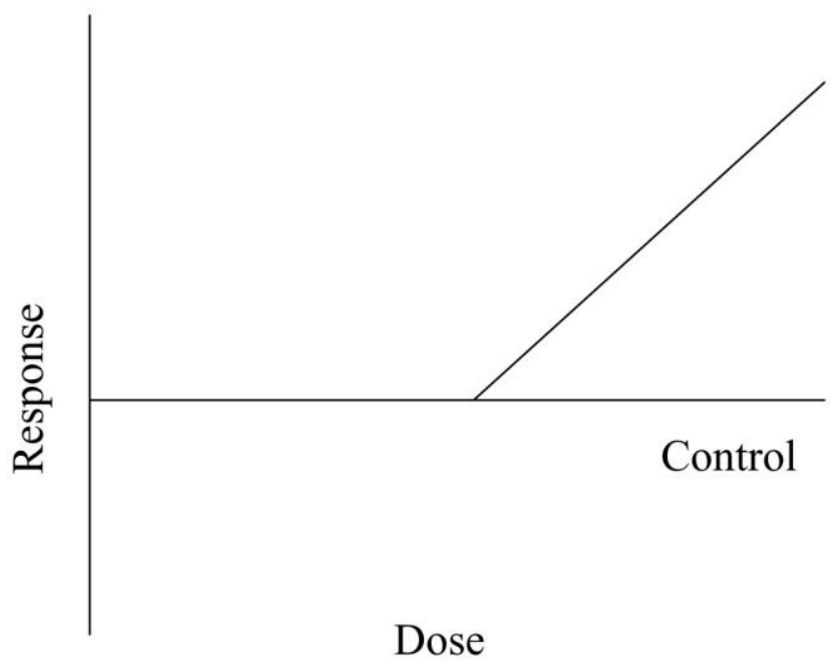

Figure 1. This curve represents a typical threshold dose-response relationship, in which a toxin begins to cause harmful effects (relative to controls) at some "threshold" dose level and then begins to cause increasingly harmful effects.

claims. ${ }^{2}$ Moreover, even if one took the very narrow view that the influences of values on the availability of scientific data and hypotheses are not a subject for epistemology, we would still insist that they merit further study because of their subtlety and complexity. In the case studies on pollution research to follow, we aim to show that these seemingly trivial influences, and the often unappreciated avenues through which they occur, can have a considerable impact on the direction a developing area of science might take.

For over 50 years, toxicologists in the United States have been attempting to establish standards for safe human exposure to hazardous substances. On the basis of experimental data collected during the nineteenth and twentieth centuries, they found that the dose-response relationship (for noncarcinogenic toxins) appeared to approach zero below some "threshold" dose level and to rise rapidly at somewhat higher dose levels (see Figure 1; Calabrese 2005). Toxicologist Edward Calabrese claims that, on the basis of these results,

2. Carl Cranor (1990) argues, e.g., that it is often socially advantageous for scientists to choose which hypotheses to accept, even though those decisions are value laden. He notes that, when scientists try to provide purely objective information and leave all value judgments up to policy makers or judges, the technical information is often misinterpreted. 


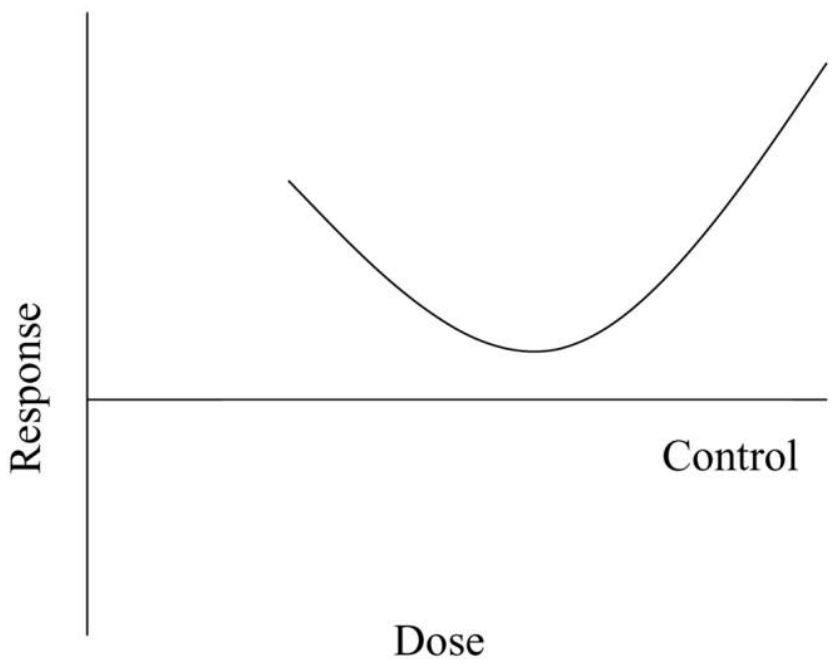

Figure 2. This curve represents a $U$-shaped dose-response relationship that might be characteristic of endocrine disruption.

the belief in the threshold model was born, that is, a belief based on experience and data. So strong was this belief in the threshold dose response model as a fundamental component of general biological process that it has subsequently ruled the fields of pharmacology and toxicology. $(2005,644)$

Despite the current dominance of the threshold model, a variety of recent research has raised questions about it. For example, some scientists claim that extremely low doses of many chemicals might mimic hormones such as estrogen and be responsible for declines in animal populations, human reproductive cancers, immune disorders, and declining male sperm counts (see, e.g., Colborn, Dumanoski, and Myers 1996; Krimsky 2000). The "endocrine-disruption" (ED) hypothesis could require significant alterations to the threshold dose-response model because it suggests that the effects of many chemicals might approach a threshold but then become increasingly harmful at lower dose levels, as shown in Figure 2 (see, e.g., Nagel et al. 1997; vom Saal and Hughes 2005). In contrast, Calabrese and Baldwin (2003) have proposed that a biological phenomenon called "chemical hormesis" is widely generalizable. Hormesis involves beneficial biological effects caused by chemicals at doses below the threshold for their harmful effects, as shown in Figure 3.

A third phenomenon that challenges the threshold model is multiple chemical sensitivity (MCS). Individuals with MCS suffer from extreme 


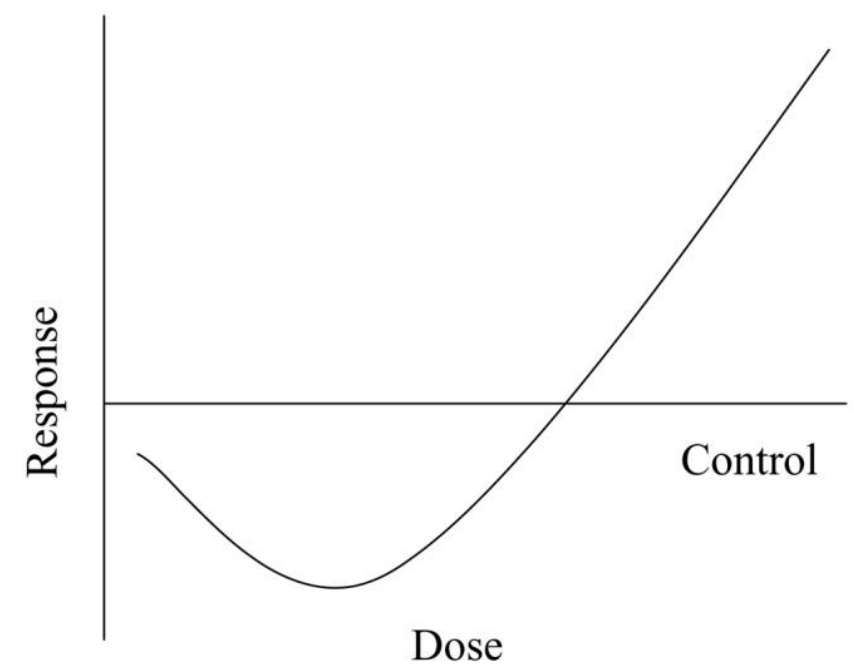

Figure 3. This curve represents a dose-response relationship characteristic of hormesis, in which a toxin's effects (relative to controls) reverse at low doses.

sensitivity to toxic chemicals. This mysterious disorder might be linked to a number of other medical problems, including "Gulf War syndrome," "sick-building syndrome," and even chronic fatigue syndrome and fibromyalgia (Ashford and Miller 1998). The phenomenon could require abandoning any standardized dose-response model, at least for MCS patients. For example, a single MCS patient might not always exhibit the same toxic effects at the same dose level, different MCS patients might exhibit completely different toxic responses to the same chemical at the same dose level, and MCS patients might display a multiplicity of toxic symptoms that are entirely different from those displayed (even at higher dose levels) by typical individuals (Staudenmayer et al. 2003).

The following sections consider three avenues through which nonepistemic values associated with discovery and pursuit have influenced appraisal of the ED, MCS, and hormesis hypotheses: (1) by guiding the choice of questions and research projects, (2) by altering experimental design, and (3) by affecting the creation and further investigation of theories or hypotheses. Nonepistemic values operating along the first two avenues of influence can cause shifts in the data available to scientists, whereas the third form of influence is based on a change in available theories.

3. Avenue 1: Choice of Questions and Research Projects. There are a mul- 
titude of ways in which nonepistemic values influenced the choice of research projects in twentieth-century pollution research. It is particularly noteworthy that these values sometimes shifted research emphasis from one question to a different but closely related question, thereby altering available data in a way that changed researchers' conclusions in a given area of inquiry. Consider, for example, that during the early to midtwentieth century, nonepistemic values discouraged the collection of data that might have challenged the dominant threshold dose-response model. The primary goal of toxicology during this time period was to identify the most obvious harmful effects of chemicals. There was therefore much more scientific interest in collecting dose-response data at high doses, at which harmful effects became apparent. The threshold model appeared to be fairly well supported at this point, given its biological plausibility and the data that supported it. In recent decades, however, a "sea change" has occurred, and several nonepistemic values are now stimulating lowdose research that threatens the threshold model. One of these factors is the desire (largely on the part of the military and the industrial sector) to weaken current environmental regulations. For example, the chemical industry and the U.S. Air Force have heavily funded Calabrese's research in support of hormesis (Kaiser 2003). Based on the newly collected data, the warrant for the threshold model has dropped.

Another effect of nonepistemic values on the collection of data in contemporary toxicology is to encourage more research on some challenges to the threshold dose-response model than on others. Many commentators have noted that it is difficult to obtain grants to study controversial hypotheses (such as MCS or hormesis or endocrine disruption) until there is a significant amount of preliminary information about them (see, e.g., Ashford and Miller 1998; Krimsky 2000; Elliott 2004). Unfortunately, it is difficult to obtain this preliminary information without having some other source of research support. This can potentially result in a skewing of research toward projects that favor the nonepistemic values of organizations that have enough money to fund speculative research projects that might serve their interests. For example, even though hormesis was hardly given any attention by the mainstream scientific community during the mid-twentieth century, the chemical industry and the Air Force were willing to start spending a significant amount of money in the 1990s to investigate it (Kaiser 2003). Frederick vom Saal (2007) goes so far as to say that industry groups were motivated to study hormesis partly because they saw it as a strategy for counteracting growing evidence from ED research that very low doses of toxins could be more harmful than previously thought. In contrast to hormesis proponents, MCS patients have struggled to find "deep pockets" with the same motivation to legitimize their alleged health problems. On the contrary, industry groups are ac- 
tually investing significant funding into the effort to show that MCS is merely a psychological phenomenon, akin to posttraumatic stress disorder (Ashford and Miller 1998).

These examples illustrate how nonepistemic values associated with the choice of questions and research projects can also influence which new hypothesis researchers accept as the successor to the threshold model. As vom Saal (2007) intimates, part of the motivation for industry and military groups to fund hormesis research was to obtain as much data as possible in favor of that hypothesis. They may have supposed that, even if some chemicals exhibit hormetic effects and other chemicals exhibit effects consistent with endocrine disruption, toxicologists would nevertheless be more inclined to accept a hormetic model as their default if most of the available information fits that hypothesis. Similarly, even if MCS is a legitimate physiological phenomenon, it is unlikely to become widely accepted if researchers cannot obtain funding to study it.

One might worry at this point that our argument is trading on ambiguities between various notions of theory appraisal, including between epistemic judgment and practical decisions about how to act. Our critics might insist that the toxicologists described in our case studies should be withholding their epistemic judgment that any particular dose model is true or accurate, because there has been inadequate evidence available. According to this perspective, any decisions to accept a particular doseresponse model should be seen as practical decisions about what models to accept for the purposes of formulating policy (see, e.g., Giere 2003; Dorato 2004; Mitchell 2004).

We doubt that this attempt to limit nonepistemic influences on theory appraisal is workable. First, there should be no doubt that, in principle, nonepistemic factors can affect epistemic assessments via the avenue that we have described. One can write a detective story such that support for the butler hypothesis given the current evidence is as high as one likes, even though introducing new evidence decisively refutes the butler hypothesis and confirms one of its rivals. How often these conditions are met in actual scientific practice is an important empirical question. Epistemologists could attempt to limit the frequency of cases in which nonepistemic factors influence epistemic judgments (as opposed to policy decisions) by drawing a sharp distinction between belief and action and setting high epistemic standards for belief. In our view, such a move would trivialize belief as a category for understanding actual scientific practice and have the consequence that most of the interesting scientific judgments would fall on the practical side of the divide.

4. Avenue 2: Designing Studies. Nonepistemic values can have much more subtle but perhaps just as powerful effects on the availability of data via 
a second avenue of influence, namely, affecting the design of studies. These influences are particularly vivid in the case of MCS, insofar as some researchers are trying to find evidence that it involves a genuine physiological disorder caused by toxic chemicals, whereas other scientists aim to show that it is a psychologically originating phenomenon akin to posttraumatic stress disorder. One strategy for determining whether MCS is "physiological" or "psychological" in origin is to perform blinded, controlled studies in which MCS sufferers are exposed either to chemicals that allegedly trigger harmful reactions or to harmless substances. Many of these studies have shown little evidence of genuine effects from the alleged trigger chemicals (Das-Munshi, Rubin, and Wessely 2006). Nevertheless, some researchers who are particularly sympathetic to MCS sufferers have been working with these patients to detect possible design flaws and to develop experiments that have a better chance of uncovering evidence for physiological aspects of the phenomenon (Ashford and Miller 1998; Joffres, Sampalli, and Fox 2005).

In contrast, those who prefer psychological interpretations of the phenomenon have designed very different studies, as highlighted by conflicts between veterans groups and the U.S. Veterans Affairs (VA) office. Although it is not clear that Gulf War syndrome (GWS) is the same as MCS, it includes many of the same features, including apparent chemical sensitivities that many skeptics attribute to psychological causes. Many veterans believe that the Research Working Group that the VA created in the 1990s was motivated by financial considerations to pursue research in a manner that would minimize evidence for physiological explanations of GWS (GAO 1998). One alleged strategy of the Research Working Group was to provide physical exams on thousands of veterans but to avoid performing tests (e.g., brain scans or genetic tests) that might provide evidence for a physiological problem. Moreover, the U.S. General Accounting Office (GAO) claims that the Department of Defense used questionable models for estimating the plume heights of chemical warfare agents released during the war, therefore underestimating the number of exposed troops (GAO 2004). In addition, the GAO claims that the VA emphasized epidemiological studies that were likely to be inconclusive (GAO 1998).

In the case of endocrine disruption, Frederick vom Saal and Claude Hughes provide another vivid example of the ways that nonepistemic values appear to affect the design of some industry studies. Regarding bisphenol A (BPA), an alleged endocrine disruptor that is used in the manufacture of many plastics, they report, "For government-funded published studies, 94 of $104(90 \%)$ report significant [biological] effects at doses of BPA $<50 \mathrm{mg} / \mathrm{kg} /$ day. No industry-funded studies ( 0 of 11 , or $0 \%)$ report significant effects at these same doses" $(2005,928)$. They argue 
that the discrepancy between these studies can be attributed to four design features that tended to produce negative results in the industry studies: "strain of experimental animal [i.e., using an especially insensitive strain], misinterpretation of finding no significant effects for the positive controls, animal feed, and specific end point examined" (928).

These examples from MCS and ED research illustrate that nonepistemic values can have a major influence on theory appraisal by affecting experimental design and consequently altering the data available to scientists. This avenue of influences is particularly interesting because of its subtlety. Although the epistemic conclusions licensed by these studies are affected by nonepistemic values, these effects are not apparent without very detailed scrutiny. One might object, however, that the cases considered here constitute examples of bad science because study designs were inappropriately manipulated. Therefore, although they may provide $d e-$ scriptive examples of how nonepistemic values have in fact affected theory appraisal, one might think that they are not relevant to philosophical discussions about how nonepistemic values can appropriately influence theory appraisal. The difficulty with this objection is that there are often no clear demarcation lines between good and bad study designs. For example, if scientists allied with industry would design a toxicology study so that it employed a robust strain of rats, whereas those allied with environmental groups would have used a particularly sensitive strain, it is not entirely clear that either group would have performed bad science. Nevertheless, their nonepistemic values could have a significant influence on whether the available evidence supports the claim that a particular chemical is harmful or not.

5. Avenue 3: Development of Theories. A third avenue through which nonepistemic values associated with discovery and pursuit affect theory appraisal is by altering available theories. For example, the ED hypothesis proved very difficult to formulate, despite significant evidence in favor of it, because it integrated data from a wide range of disciplines. Theo Colborn, the central figure in developing the hypothesis, persevered in developing the hypothesis in part because she was influenced by nonepistemic values of deep concern for the environment.

We see similar dynamics in the hormesis case. Although the hormesis hypothesis had been proposed by the beginning of the twentieth century, it languished in obscurity for decades. It arguably would have remained that way if industry-oriented scientists had not seized on the hypothesis and popularized it. Once the hormesis and ED hypotheses were available, it became clear that a variety of data supported them. It remains unclear precisely what proportion of chemicals (under which conditions) produce these phenomena, but the hypotheses clearly have some legitimacy that 
could easily have gone unnoticed if scientists had not pushed them forward for nonepistemic reasons.

This third avenue of influences was previously emphasized by Kathleen Okruhlik (1994). She argued that masculine values played a significant role in the acceptance of theories such as the "Sleeping Beauty/Prince Charming" model of the egg and sperm or the man-the-hunter view of human evolution. Nevertheless, she (like us) did not claim that these nonepistemic values altered the evidential relationship between the available theories and data. Rather, she claimed that gender-based values strongly influenced which theories were proposed. Because she insisted that researchers ultimately accept whichever available theory is best supported by current evidence, she argued that nonepistemic values could have a significant influence on the cognitive content of science by determining which theories are available to compete.

Although we agree with much of Okruhlik's analysis, we want to distance ourselves from her "comparative model" of theory appraisal. Her critics might argue, for example, that scientists are justified in accepting a theory only if it meets a particular standard of evidence, not just because it is the best one available (see, e.g., Shrader-Frechette 2006). We believe that our third avenue of nonepistemic influences remains unaffected by this objection, because the effects that we observe in the ED and hormesis cases do not depend on a comparative model of theory appraisal. These hypotheses hold up not because they are the best available but rather because they are well supported by current evidence. The reason is partly that, as Okruhlik herself pointed out, the proposal of new hypotheses can transform what appeared to be irrelevant facts into crucial pieces of evidence. For example, what formerly appeared to be unimportant and presumably erroneous stimulatory low-dose data points in previous toxicology studies suddenly became crucial pieces of evidence after the hormesis hypothesis was formulated. Similarly, what had previously appeared to be completely unrelated anomalous phenomena (e.g., loafing behavior in bald eagles, low hatching and survival rates for alligator eggs, and reproductive cancers in humans whose mothers took the synthetic estrogen DES) suddenly constituted a tightly knit web of related evidence after Colborn developed the ED hypothesis.

6. Conclusion. We have examined three avenues through which nonepistemic values associated with scientific discovery and pursuit affect theory appraisal: (1) guiding the choice of questions and research projects, (2) altering experimental design, and (3) affecting the creation and further investigation of theories or hypotheses. Our claims about these three avenues rest on the uncontroversial principle that the evidential support for a theory varies depending on the data that have been collected and the 
other theories that are under consideration. We have seen that this straightforward point about theory confirmation takes on a new significance when one looks at detailed case studies from a field such as pollution research. Nonepistemic values can subtly influence the evidential support for a theory by altering the sorts of data that scientists collect within a particular area of scientific inquiry, and they can transform seemingly irrelevant findings into crucial pieces of evidence.

The significance of our analysis lies partly in the support that it provides for studies in the social epistemology of science (see, e.g., Kitcher 2001; Longino 2002). To the extent that nonepistemic values associated with discovery and pursuit can influence our epistemic appraisal of theories in subtle and underappreciated ways, it seems all the more important to reflect on how to manage these values. Numerous commentators are expressing dismay over the current amount of research funding originating with private organizations that have vested interests in the outcome of the research (e.g., Shrader-Frechette 2007; Elliott 2008). Pharmaceutical companies alone spent $\$ 32$ billion for research and development in 2002, which was more than the entire National Institutes of Health operating budget of $\$ 24$ billion for that year. ${ }^{3}$ Some reports suggest that, for every $\$ 6$ that private interests spend on scientific research, the federal government funds only about $\$ 1$ of nonmilitary research (Shrader-Frechette 2007). Thus, this paper encourages the growing efforts to reflect on both the ethical and the epistemic ramifications of increasingly privatized scientific research funding.

\section{REFERENCES}

Ashford, Nicholas, and Claudia Miller (1998), Chemical Exposures: Low Levels and High Stakes. 2nd ed. New York: Van Nostrand Reinhold.

Calabrese, Edward (2005), "Historical Blunders: How Toxicology Got the Dose-Response Relationship Half Right", Cellular and Molecular Biology 51: 643-654.

Calabrese, Edward, and Linda Baldwin (2003), “Toxicology Rethinks Its Central Belief”, Nature 421: 691-692.

Colborn, Theo, Dianne Dumanoski, and Jonathan Myers (1996), Our Stolen Future. New York: Dutton.

Cranor, Carl (1990), "Some Moral Issues in Risk Assessment", Ethics 101: 123-143.

Das-Munshi, Jayati, James Rubin, and Simon Wessely (2006), "Multiple Chemical Sensitivities: A Systematic Review of Provocation Studies", Journal of Allergy and Clinical Immunology 118: 1257-1264.

Dorato, Mauro (2004), "Epistemic and Nonepistemic Values in Science", in Machamer and Wolters 2004, 52-77.

Douglas, Heather (2000), "Inductive Risk and Values in Science", Philosophy of Science 67: 559-579.

Elliott, Kevin (2004), "Error as Means to Discovery”, Philosophy of Science 71: 1-24.

3. See the Columbia University Responsible Conduct of Research Web site's module on conflicts of interest (http://ccnmtl.columbia.edu/projects/rcr/index.html). 
(2008), "Scientific Judgment and the Limits of Conflict-of-Interest Policies", $A c$ countability in Research: Policies and Quality Assurance 15: 1-29.

GAO (General Accounting Office) (1998), Gulf War Illnesses: Federal Research Strategy Needs Reexamination. Washington, DC: GAO. Available at http://www.gao.gov/archive/ 1998/ns98104t.pdf.

- (2004), Gulf War Illnesses: DOD's Conclusions about U.S. Troops' Exposure Cannot Be Adequately Supported. Washington, DC: GAO. Available at http://www.gao.gov/ new.items/d04159.pdf.

Giere, Ronald (2003), “A New Program for Philosophy of Science?", Philosophy of Science 70: $15-21$.

Howard, Don (2006), "Lost Wanderers in the Forest of Knowledge: Some Thoughts on the Discovery-Justification Distinction", in J. Schickore and F. Steinle (eds.), Revisiting Discovery and Justification: Historical and Philosophical Perspectives on the Context Distinction. New York: Springer, 3-22.

Jeffrey, Richard (1956), "Valuation and Acceptance of Scientific Hypotheses", Philosophy of Science 23: 237-246.

Joffres, M., T. Sampalli, and R. Fox (2005), "Physiologic and Symptomatic Responses to Low-Level Substances in Individuals with and without Chemical Sensitivities: A Randomized Controlled Blinded Pilot Booth Study", Environmental Health Perspectives 113: $1178-1183$

Kaiser, Jocelyn (2003), “Sipping from a Poisoned Chalice”, Science 302 (October 17): 376 379.

Kitcher, Philip (2001), Science, Truth, and Democracy. Oxford: Oxford University Press.

Krimsky, Sheldon (2000), Hormonal Chaos: The Scientific and Social Origins of the Environmental Endocrine Hypothesis. Baltimore: Johns Hopkins University Press.

Kuhn, Thomas (1977), "Rationality, Value Judgment, and Theory Choice", in The Essential Tension. Chicago: University of Chicago Press, 320-339.

Laudan, Larry (1977), Progress and Its Problems. Berkeley: University of California Press. (1984), Science and Values. Berkeley: University of California Press.

Longino, Helen (1990), Science as Social Knowledge. Princeton, NJ: Princeton University Press. (2002), The Fate of Knowledge. Princeton, NJ: Princeton University Press

Machamer, P., and G. Wolters, eds. (2004), Science, Values, and Objectivity. Pittsburgh: University of Pittsburgh Press.

Mitchell, Sandra (2004), "The Prescribed and Proscribed Values in Science Policy", in Machamer and Wolters 2004, 245-255.

Nagel, S., F. vom Saal, K. Thayer, M. Dhar, M. Boechler, and W. Welshons (1997), "Relative Binding Affinity-Serum Modified Access (RBA-SMA) Assay Predicts the Relative In Vivo Activity of the Xenoestrogens Bisphenol A and Octylphenol", Environmental Health Perspectives 105: 70-76.

Okruhlik, Kathleen (1994), "Gender and the Biological Sciences", Canadian Journal of Philosophy 20 (suppl.): 21-42.

Rudner, Richard (1953), "The Scientist Qua Scientist Makes Value Judgments", Philosophy of Science 20: 1-6.

Ruphy, Stephanie (2006), “Empiricism All the Way Down': A Defense of the Value-Neutrality of Science in Response to Helen Longino's Contextual Empiricism", Perspectives on Science 14: 189-214.

Shrader-Frechette, Kristin (2006), "Comparativist Philosophy of Science and Population Viability Assessment in Biology: Helping Resolve Scientific Controversy", Philosophy of Science 73 (December): 817-828. (2007), Taking Action, Saving Lives. Oxford: Oxford University Press.

Staudenmayer, H., K. Binkley, A. Leznoff, and S. Phillips (2003), "Idiopathic Environmental Intolerance: Part 1: A Causation Analysis Applying Bradford Hill's Criteria to the Toxicogenic Theory", Toxicological Reviews 22: 235-246.

vom Saal, F. (2007), "Hormesis Controversy", Environmental Science and Technology 41: 3. 
vom Saal, F., and C. Hughes (2005), "An Extensive New Literature Concerning Low-Dose Effects of Bisphenol A Shows the Need for a New Risk Assessment", Environmental Health Perspectives 113: 926-933. 